\title{
Orodental Care related to Radiotherapy for Head and Neck Cancer
}

\author{
R Atri, AK Dhull, V Nair, R Dhankhar, V Kaushal
}

\section{ABSTRACT}

Orodental care is mandatory in radiotherapy for head and neck cancer patients. Radiation therapy of malignant tumors of head and neck is associated with number of side effects, which can lead to acute and chronic oral complications. It is important to prevent and treat orofacial complications which require a multidisciplinary approach. All patients should undergo oral screening before radiotherapy for tumors of head and neck. Many oral conditions, such as poor oral hygiene, broken teeth, defective restorations and periodontal disease, are likely to precipitate complications during and after a course of radiation therapy and it is always better to deal with these problems before starting radiotherapy. The risk of osteoradionecrosis will be reduced to zero if there was a 21 -day or greater interval between extraction and initiation of radiation therapy. Systematically applied oral hygiene protocols during radiotherapy may reduce the incidence, severity and duration of oral complications. It is well accepted that tooth extraction should be avoided even after many years of head and neck radiotherapy. If required it should be done by an experienced dental surgeon with proper prophylactic measures. The dental professional plays a relevant role in the prevention and healing or control of the oral complications. The relief of symptoms and the eradication of clinical manifestations largely improve patient quality of life.

\section{Contact Author}

Dr. Rajeev Atri

E-mail : rajeevatri@gmail.com

Keywords: Radiotherapy, Oral Hygiene, Osteoradionecrosis

$\mathrm{H}$ ead and neck cancers constitute a major proportion of regional malignancies in India. "Head and Neck Cancer" usually refers to neoplasms arising from below the skull base to the region of thoracic inlet.(1) The anatomy and physiology of this region are uniquely complex, and the function and appearance are critical to patient's selfimage and quality of life. Most patients with head and neck squamous cell carcinoma are middle aged adult males in lower socio-economic classes who are chronic tobacco and alcohol consumers and have advanced tumors. These patients therefore tend to be less health conscious and to have less social support than most other cancer patients. Options for treatment of head and neck cancers are surgery, radiotherapy and chemotherapy.
Most head and neck cancer patients are treated with high-dose radiotherapy to large irradiation fields encompassing sensitive structures, including the oral cavity and surrounding structures. Radiation therapy of malignant tumors of head and neck is associated with number of side effects, which can lead to oral complications. The preservation of form and function of these structures are the key elements in over all management of head and neck cancer patients. It is Important to prevent and treat orofacial complications which require an multidisciplinary approach, that includes a dedicated team of radiation oncologist, head and neck surgeon, dentist, nurse, dietician, physical therapist, social worker and in some instances plastic surgeon, prosthodontist, and psychologist. 


\section{Radiation Induced Complications Acute Complications}

A therapeutic dose of radiation in head and neck cancer usually comprises a total of $64 \mathrm{~Gy}$ to $70 \mathrm{~Gy}$ in 32 to 35 fractions with daily dose of 1.8 to $2.0 \mathrm{~Gy}$ per fraction. Acute complications appear one to two weeks after starting the patient on radiation treatment. Oral mucositis, alteration or loss of taste (Dysgeusia), infection (bacterial, fungal \& viral), change in salivary composition, periodontium pain are common acute complications which subside two to four weeks after completion of radiotherapy.(2)

\section{Chronic Complications}

The long-term effects are due to changes in the vascularity and cellularity of soft tissue and bone, damage to salivary glands and increased collagen synthesis, leading to hypovascularity, hypocellularity and hypoxia of the tissues. The common chronic complications are: xerostomia, dental alterations, rampant caries, demineralization, alteration or loss of taste (Dysgeusia), secondary opportunistic infections (e.g. candidiasis), oral ulceration (soft tissue necrosis), osteoradionecrosis, pain, temporomandibular disorders/ trismus, nutritional deficiencies, psychosocial impact. To a significant degree, the oral problems associated with radiation therapy can be prevented or minimized through optimal management.(2)

\section{Orodental Care before Radiotherapy}

A consultation with a dental team experienced in caring for patients undergoing treatment for head and neck cancer should be completed before the start of therapy. Establishment and maintenance of oral health, careful dental treatment planning is essential to minimize oral disease and possible adverse consequences. All patients should undergo oral screening before radiotherapy for tumors of head and neck.(1) The screening includes complete dental evaluation from dental surgeon with full description of the radiation treatment. The clinical examination should include extra oral features, particularly, lymph nodes, metastasis and mouth opening followed by comprehensive intraoral examination. This includes a record of dentition, presence of caries, periodontal disease, swelling fistula and oral hygiene. It is always beneficial to achieve a good level of oral hygiene before radiotherapy. In addition to the clinical examination, a thorough radiographic examination is crucial to determine the presence of inflammatory periapical abnormalities, periodontal status, other dental disease and tumour invasion of bone. A panoramic radiograph plus selective periapical or bitewing films (or both) should be done for preradiotherapy dental assessments.(3) Many oral conditions, such as poor oral hygiene, broken teeth, defective restorations and periodontal disease, are likely to precipitate complications during and after a course of radiation therapy. Dental and periodontal disease should be treated, oral infections should be controlled prior to starting radiotherapy treatment in head and neck cancers. $(4,5)$

\section{Criteria for Preradiotherapy Extractions}

The criteria are: Caries (nonrestorable), active periapical disease (symptomatic teeth), moderate to severe periodontal disease, lack of opposing teeth, compromised hygiene, partial impaction or incomplete eruption, low dental awareness and lack of cooperation.(6) Pre-irradiation extractions, when performed and timed correctly, do not significantly increase the overall risk of osteoradionecrosis. Frequently suggested healing intervals ranged from 10 to 14 days. The risk is reduced to zero if there was a 21-day or greater interval between extraction and initiation of radiation therapy.(5,6) All major surgery should be performed 4-6 weeks in advance of start of radiotherapy.

\section{Orodental Care during Radiotherapy}

Monitoring of the oral cavity should be increased during radiation therapy in an effort to decrease the severity of side effects. Systematically applied oral hygiene protocols may reduce the incidence, severity and duration of oral complications.(7) It is imperative that patients continue oral hygiene regimen throughout their course of cancer therapy.(8) The orofacial complications of cancer therapy depend mainly on the treatment modality used and to some extent also on host factors. For example, the severity of mucositis following oral radiation therapy depends upon, the type of radiation used, the rate at which it is delivered, the total dose given, individual response to the therapy and factors such as use of tobacco and alcohol products. Dietary control and topical fluoride therapy are essential and should be started on the first day of radiation therapy, continued for life or the duration of Xerostomia.(9) Maintenance of good oral hygiene- brushing 2 to 4 times daily with soft-bristled tooth brush and fluoride toothpaste or gel to help prevent plaque accumulation and demineralization or caries of the teeth; flossing daily is of great help.(9) Almost all patients undergoing head and neck radiation therapy experience confluent mucositis by approximately the third week of treatment. Maintaining a self-care regimen may decrease the incidence. The discomfort of mucositis is reduced with, coating agents, topical anesthetics, analgesics and systemic analgesics. In general, mucositis should be treated conservatively. Further tissue irritation and factors damaging epithelium, plaque control and oral hygiene should be maintained with tooth brushing, a soft bland diet, avoiding irritants such as tobacco, alcohol or spices. Maintenance of adequate hydration and nutrition is helpful. Jaw exercises (mechanotherapy) may limit the severity of trismus, but they will not mobilize fibrosis once it has occurred.(10-13) Health care providers are concerned about oral infections, preventing local and systemic infections in addition to managing oral symptoms. Treating infections as soon as they are detected is helpful to reduce pain as well as the spread of infection. A fungal, bacterial or viral culture is 
recommended if infection is suspected. Frequent ingestion of small amounts of food (every 1-2 hours), ingestion of foods containing abundant calories and proteins is recommended. Stimulate appetite with light exercise, creative menus, avoidance of strong aromas.(14)

\section{Orodental Care Post Therapy}

Interventions that may be beneficial following treatment include: clean teeth and gums after meals and before sleep with tooth brush or swab as tolerated; rinse the mouth with water regularly; if dentures are worn, remove and clean them daily and leave out while at rest; avoid painful stimuli such as hot food and drinks, spicy food, alcohol and smoking; regular inspection of mouth by the patient and health professionals; report any redness, tenderness or sores on the lips or in mouth; provide comfort measures such as lubrication of the lips, topical anesthesia and analgesics; prompt treatment of mucositis symptoms and oral infections. Long-term management and close follow up of patients after radiation therapy is mandatory. Continue to follow an oral health self-care regimen to keep the teeth and gums healthy, facilitate repair of any residual oral damage.(15) The patient should attend additional dietary counseling sessions. The referrals of patients to support groups are useful adjunct to patients' return to optimal functioning. Close follow-up will facilitate the management of any chronic complications that may occur. It is well accepted that tooth extraction should be avoided even after many years of head and neck radiotherapy and complete or removable dentures should be avoid for one year. Decreased local vascularization and its consequent radiation impaired healing capacity, particularity in the mandible, is the main cause of osteoradionecrosis. A higher incidence of osteoradionecrosis is observed after cumulative radiation doses to the bone exceed $65 \mathrm{~Gy}$. Some studies have reported a two-fold increased risk of necrosis if teeth were extracted after radiotherapy compared with pre-irradiation therapy dental extractions.(15) Also, antibiotic coverage is strongly recommended. There is some evidence that hyperbaric oxygen treatment is more beneficial than conventional antibiotic prophylaxis in preventing osteoradionecrosis. Mechanotherapy for trismus, treatment of inflammations, mucositis and xerostomia should be undertaken promptly.(15)

\section{Conclusion}

The orodental care plays an important part in management of head and neck cancer patients. The complications of radiotherapy in head and neck cancers must be considered thoroughly-every effort is undertaken to minimize the oral morbidity before, during and after cancer treatment and throughout the patient's lifetime. The dental professional plays a relevant role in the prevention and healing or control of the oral complications. The relief of symptoms and the eradication of clinical manifestations largely improve patient quality of life.

\section{THE AUTHORS}

\section{Dr. Rajeev Atri}

MD

Senior Resident

Department of Radiotherapy, Regional Cancer Centre, PGIMS, Rohtak, Haryana, India.

EMAIL: rajeevatri@gmail.com

\section{Dr. Anil Kumar Dhull}

MBBS

Junior Resident

Department of Radiotherapy, Regional Cancer Centre, PGIMS, Rohtak, Haryana, India.

\section{Dr. Vimoj Nair}

MBBS

Junior Resident

Department of Radiotherapy, Regional Cancer Centre,

PGIMS, Rohtak, Haryana, India.

\section{Dr. Rakesh Dhankhar}

MD

Assistant Professor

Department of Radiotherapy, Regional Cancer Centre, PGIMS, Rohtak, Haryana, India.

\section{Dr. Vivek Kaushal}

$\mathrm{MD}, \mathrm{DNB}$

Senior Professor and Head

Department of Radiotherapy, Regional Cancer Centre,

PGIMS, Rohtak, Haryana, India.

\section{References}

1. Mohanti BK, Bahadur S, Lal P, Gairola M, Rath GK. Cancers of the head and neck. In: Rath GK, Mohanti BK, editors. Textbook of Radiation Oncology, Principles and Practice. New Delhi; B.I. Churchill Livingstone, 2000: 131-99.

2. Specht L Oral complications of head and neck radiation patients, introduction and scope of problem. Support Care Cancer 2002;1036-39.

3. Tugnait A, Clerehugh V, Hirschmann PN. The usefulness of radiographs in diagnosis and management of periodontal diseases: a review. J Dent 2000;28:219-26.

4. Jansma J, Vissinik A, Bouma J. A survey of prevention and treatment regimen for oral sequele resulting from head and neck radiation therapy used in Dutch radiotherapy institutes. Int J Radiat Oncol Biol Phys 1992;24:359-67.

5. Jansma J, Vissink A, Spijkervet FKL, Panders AK, Vermey A, Roodenburg JLN, et al. Protocol for the prevention and treatment of oral complications of head and neck radiotherapy. Cancer 1992;70: 2171-80.

6. Beumer J, Harrison R, Sanders B, Kurrassch M. Preradiation dental extraction and the incidence of bone necrosis. Head Neck Surg 1983;5:514-21.

7. Turhal NS, Erdal S, Karacay S. Efficacy of treatment to relieve mucositis-induced discomfort. Support Care Cancer. 2000;8(1):5558.

8. Barasch A, Safford MM. Management of oral pain in patients with malignant diseases. Compendium 1993;14(11):1376,1378-82.

9. Katz S. The use of fluoride and chlorhexidine for the prevention of radiation caries. J Am Den Assoc 1982;104:164-70.

10. Foote RL, Loprinizi CL, Frank AR, O'Fallon JR, Gulavita S, Twefik $\mathrm{HH}$, et al. Randomized trial of a chlorhexidine mouthwash for alleviation of radiation-induced mucositis. J Clin Oncol 1994;12 (12):2630-33. 
11. Epstein JB, Silverman S, Paggiarino DA, Crocket S, Schubert MM, Senzer NN, et al. Benzydamine $\mathrm{HCl}$ for prophylaxis of radiation inducedoral mucositis: results from a multicenter, randomized, double blind, placebo-controlled clinical trial. Cancer 2001;92 (4):875-85.

12. Whitmyer CC, Waskowski JC, Iffland HA. Radiotherapy and oral sequelae: preventive and management protocols. J Dent Hyg 1997;71(1):23-29.
13. Cremonese G, Bryden G, Bottcher C. A multidisciplinary team approach to preservation of quality of life for patients following oral cancer surgery. OHL Head Neck Nurs 2000;18(2):6-11.

14. Jham BC, Freire ARS. Oral complications of radiotherapy in the head and neck. Rev Bras Otorrinolaringol 2006;72(5):704-08.

15. Vissink A, Burlag FR, Spijkervet FKL, Jansm J, Coppes RP. Revention and treatment of the consequences of head and neck radiotherapy. Crit Rev Oral Biol Med 2003;14(3):213-25. 